\title{
Secure DS-CDMA Spreading Codes using Fully Digital Multidimensional Multiscroll Chaos
}

\author{
A. S. Mansingka ${ }^{1, *}$, M. Affan Zidan ${ }^{1}$, A. G. Radwan ${ }^{2}$ and K. N. Salama ${ }^{1}$ \\ ${ }^{1}$ Electrical Engineering, King Abdullah University of Science and Technology (KAUST), Thuwal, Saudi Arabia \\ ${ }^{2}$ Department of Engineering Mathematics, Faculty of Engineering, Cairo University, Egypt \\ Email: khaled.salama@kaust.edu.sa
}

\begin{abstract}
This paper introduces a generalized fully digital hardware implementation of 1-D, 2-D and 3-D multiscroll chaos through sawtooth nonlinearities in a 3rd order ODE with the Euler approximation, wherein low-significance bits pass all NIST SP. 800-22 tests. The low-significance bits show good performance as spreading code for multiple-access DS-CDMA in AWGN and multipath environments, equivalent to Gold codes. This system capitalizes on complex nonlinear dynamics afforded by multiscroll chaos to provide higher security than conventional codes with the same BER performance demonstrated experimentally on a Xilinx Virtex 4 FPGA with logic utilization less than $1.25 \%$ and throughput up to 10.92 Gbits/s.
\end{abstract}

\section{INTRODUCTION}

Chaos generation has attracted interest specifically in communication systems [1]-[8]. In particular, multi-scroll chaos is well understood [9]-[14] and has been realized through analog circuits [13], [15] but the limited dynamic range of analog components and low supply voltages has restricted the number of scrolls that can be realized [16] unless mixed-signal approaches are used [17]. Digital design enables reliable and fully digital chaotic oscillators [14], [18] for direct application in digital systems. Jerk-equation based chaotic systems [19] have been digitally implemented and assessed for the effect of bus width [18], optimized for random number generation [20], [21] and have been evaluated for different numerical techniques [22]. Furthermore, the difficulty in physical realization of complicated algebraic equations in analog form [12] motivates the digital implementation of multiscroll chaos.

Particularly, chaos-based communication is considered advantageous because chaotic signals are inherently unpredictable, wideband and have low cross-correlation, thus enabling multiple users in a spread-spectrum communication environment [1]. In particular, multiple-access differential chaosshift keying (DCSK) [2], [3] has been motivated due to the difficulty in replicating the chaotic source at the receiver. However, this approach is intrinsically insecure and inefficient as the reference chaotic sample is transmitted in addition to the modulated signal. Coherent multiple access chaos-shift keying (CSK) in direct-sequence spread-spectrum is more secure [4] and can be run at lower spreading factors. Fully digital multiscroll chaos can provide repeatable chaotic spreading codes while also having complex dynamical behavior that provides security over communication channels [12]. Chaotic

*A. S. Mansingka is currently with Oracle Corporation, Redwood, CA, USA spreading sequences surpass m-sequences and Gold codes [23] and it is already well-known that fully orthogonal Walsh codes perform very poorly in a multipath environment because of poor cross-correlation properties under delay [24].

This paper introduces a generalized hardware implementation of multidimensional multiscroll chaos with an application as spreading code in DS-CDMA by concatenating the lowsignificance bits of the three dimensional output to generate a sequence that passes all the NIST SP. 800-22 tests for statistical randomness. Extension of scrolls to higher dimensions increases number of chaotic modes to accommodate larger number of users. The resulting chaotic spreading code sources are experimentally verified on a Xilinx Virtex 4 FPGA with logic utilizations less than $1.25 \%$ and throughput up to 10.92 Gbits/s. The implemented system provides 512 distinct code streams with low cross-correlation and is easily scalable based on implementation parameters. The spreading code shows strong performance in a multiple-access environment with additive white Gaussian noise (AWGN) and multipath channels, equivalent to Gold codes while simultaneously providing security through aperiodic and complex nonlinear dynamics that pass tests for statistical randomness.

\section{Digital Implementation}

Generalized multidimensional multiscroll chaos can be described using a 3rd order ODE in $\{X, Y, Z\}$ using a sawtooth nonlinearity $F(X)$ with integer lower bound $L$, upper bound $U$ and floor function $\lfloor X\rfloor$ given by:

$$
F(X)= \begin{cases}X-U-0.5, & X \in(U+0.5, \infty) \\ X-L-0.5, & X \in(-\infty, L+0.5) \\ X-\lfloor X\rfloor-0.5, & {[L+0.5, U+0.5]}\end{cases}
$$

Essentially, multidimensional scrolls arise from expanding the nonlinearity in $X$ [17] to $Y$ and $Z$. Table I species the functions that describe $\{\dot{X}, \dot{Y}, \dot{Z}\}$ for 1-D, 2-D and 3-D multiscrolls along with the equilibrium points and Jacobian matrices at those points. All three systems have the same Jacobian, and are dissipative because the trace of the Jacobian (sum of the eigenvalues) is negative. They also have the same characteristic equation at each equilibrium point:

$$
s^{3}+\alpha s^{2}+\alpha s+\alpha=0
$$

giving eigenvalues of $(-0.9374,0.0312, \pm 0.9657 i)$ for $\alpha=$ 0.875 , with one negative real eigenvalue and a pair of complex 
TABLE I

1-D, 2-D AND 3-D MULTISCROLL CHAOS.

\begin{tabular}{|c|c|c|c|c|c|c|}
\hline & System ODE & Equilibrium Points $\left(X^{*}, Y^{*}, Z^{*}\right)$ & \multicolumn{3}{|c|}{$\operatorname{Jacobian}(j)$} & $\operatorname{Trace}(j)$ \\
\hline $1-\mathrm{D}$ & $\begin{array}{c}\dot{X}=Y \\
\dot{Y}=Z \\
\dot{Z}=-\alpha[F(X)+Y+Z]\end{array}$ & $\begin{array}{l}(i+0.5,0,0) \\
i \in\left[L_{x}, U_{x}\right)\end{array}$ & {$\left[\begin{array}{c}0 \\
0 \\
-\alpha\end{array}\right.$} & $\begin{array}{c}1 \\
0 \\
-\alpha\end{array}$ & $\begin{array}{c}0 \\
1 \\
-\alpha\end{array}$ & $-\alpha$ \\
\hline 2-D & $\begin{array}{c}\dot{X}=F(Y) \\
\dot{Y}=Z \\
\dot{Z}=-\alpha[F(X)+F(Y)+Z]\end{array}$ & $\begin{array}{c}(i+0.5, j+0.5,0) \\
i \in\left[L_{x}, U_{x}\right), j \in\left[L_{y}, U_{y}\right)\end{array}$ & {$\left[\begin{array}{c}0 \\
0 \\
-\alpha\end{array}\right.$} & $\begin{array}{c}1 \\
0 \\
-\alpha\end{array}$ & $\begin{array}{c}0 \\
1 \\
-\alpha\end{array}$ & $-\alpha$ \\
\hline $3-\mathrm{D}$ & $\begin{array}{c}\dot{X}=F(Y) \\
\dot{Y}=F(Z) \\
\dot{Z}=-\alpha[F(X)+F(Y)+F(Z)]\end{array}$ & $\begin{array}{c}(i+0.5, j+0.5, k+0.5) \\
i \in\left[L_{x}, U_{x}\right), j \in\left[L_{y}, U_{y}\right), k \in\left[L_{z}, U_{z}\right)\end{array}$ & $\begin{array}{c}0 \\
0 \\
-\alpha\end{array}$ & $\begin{array}{c}1 \\
0 \\
-\alpha\end{array}$ & $\begin{array}{c}0 \\
1 \\
-\alpha\end{array}$ & $-\alpha$ \\
\hline
\end{tabular}

conjugate eigenvalues with positive real parts. This makes all the equilibrium points of the resulting system saddle points of index 2 that are necessary for the formation of scrolls and are verified through numerical simulation. The Euler approximation (with step size $h$ ) gives the numerical solution according to Fig. 1:

$$
\begin{gathered}
X_{t+h}=X_{t}+h\left[\dot{X}_{t}\right]=P\left(X_{t}, Y_{t}\right) \\
Y_{t+h}=Y_{t}+h\left[\dot{Y}_{t}\right]=Q\left(Y_{t}, Z_{t}\right) \\
Z_{t+h}=Z_{t}+h\left[\dot{Z}_{t}\right]=R\left(X_{t}, Y_{t}, Z_{t}\right)
\end{gathered}
$$

Registers $\{X, Y, Z\}$ store the system state while combinational logic in $\{P, Q, R\}$ determines the next state. To optimize throughput, the nonlinear function(s) are pre-computed and stored in temporary register(s). A 32-bit fixed-point two's complement representation is used with 4 bits for the sign and integer part and 28 bits for the fraction part. The stepsize is $h=2^{-3}$, simplifying to a hardcoded right-shift. The system parameter is $\alpha=0.875$ and is realized through a single subtractor with $0.875 A=A-2^{-3} A$.

While many parameters can be made controllable in a digital multiscroll system [25], using only the number of scrolls in 1-D, 2-D or 3-D minimizes hardware and maximizes performance. Of particular interest is the hardware implementation of the sawtooth function in (1), shown in Fig. 2 with the resulting output in Fig. 3. $L$ and $U$ are integer-valued, with $L=-6$ fixed and a controllable 3-bit $U(U \in[-4,3])$, one less than the integer width to prevent overflow. Sign-extension by 1 bit matches $U$ with the integer width and one-padding to the right by 1 bit adds 0.5 without hardware. This is fully scalable and more control can be added simply by using a wider integer width (thus wider $L$ and $U$ ) to accommodate more scrolls. Equilibrium points of the chaotic system are

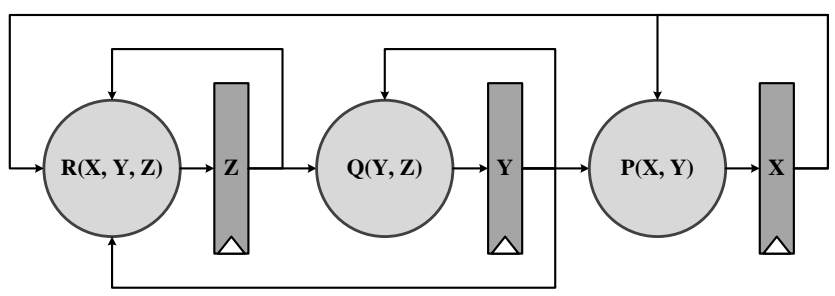

Fig. 1. Generalized schematic for multidimensional multiscroll chaos.

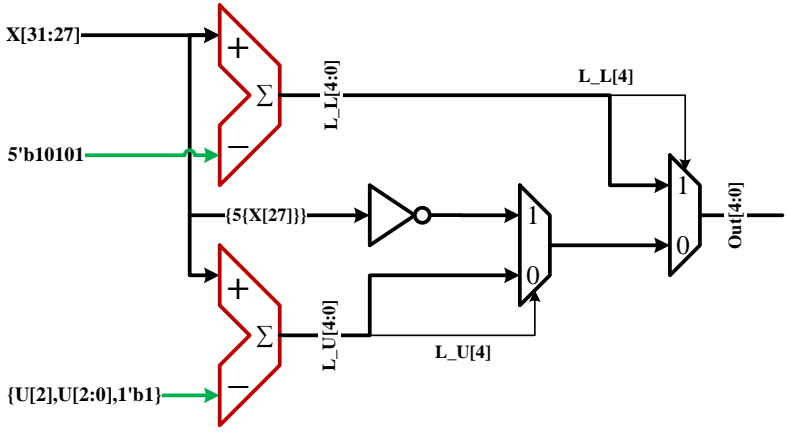

Fig. 2. Digital realization of the sawtooth function.

calculated by finding $(\dot{X}, \dot{Y}, \dot{Z})=(0,0,0)$ and are shown in Table I and give the number of scrolls in any dimension as:

$$
N_{d}=U_{d}-L_{d}+1=U_{d}+7, \quad U_{d} \in[-4,3]
$$

ranging between 3 and 10 and dependent only on the upper bound of the sawtooth nonlinearity in that dimension, giving the total number of scrolls in 1-D, 2-D and 3-D cases as:

$$
\begin{gathered}
N_{1 D}=\left(U_{x}+7\right) \\
N_{2 D}=\left(U_{x}+7\right) \times\left(U_{y}+7\right) \\
N_{3 D}=\left(U_{x}+7\right) \times\left(U_{y}+7\right) \times\left(U_{z}+7\right)
\end{gathered}
$$

\section{Chaotic Response}

Oscilloscope traces of the attractors (X-Y-Z phase plots) from the digitally implemented 1-D, 2-D and 3-D multiscroll

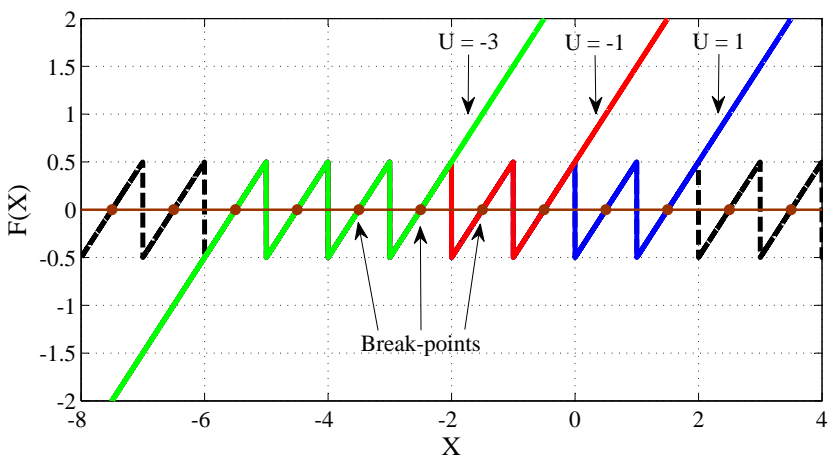

Fig. 3. The modified sawtooth function from (1) with $L=-6$ and $U=-3$, $U=-1$ and $U=1$. The original sawtooth function is shown in dashed lines. 
systems are shown in Fig. 4, indicating chaotic characteristics upon visual inspection. Finite precision numerical solutions give pseudo-chaotic trajectories that approximate the continuous-time chaotic trajectories of the ODE. However, a positive maximum Lyapunov exponent (MLE) verifiers the existence of chaotic dynamics. Mathematically, $\lambda$ (the MLE) expresses the effect of an arbitrarily small change in initial conditions on the long-term divergence in the output solution according to $\delta S(t) \approx e^{\lambda t} S(\delta t)$. Software based on [26] enables the estimation of the continuous-time MLE from discrete data. The resulting positive MLEs of $0.078,0.122$ and 0.162 for 1 -D ( 5 scrolls), 2 -D $(5 \times 5$ scrolls $)$ and 3 -D $(5 \times 5 \times 3$ scrolls) multiscroll systems verify chaotic dynamics.

\section{Implementation as Spreading Sequences:}

In all three systems, the high 8 bits from each of $\{X, Y, Z\}$ are discarded. The remaining bits together form a spreading sequence source such that the resulting 72-bit parallel output is taken serially from the most to least significant bits, leading to a single bitstream for each set of control parameters. The NIST SP. 800-22 [27] test results, area utilization and throughput on a Xilinx Virtex 4 XC4VSX35-10FF668 FPGA (30,720 LUTs, 30,720 flip-flops) are shown in Table II using 72 sets of 5,000,000 iterations. Throughput is experimentally verified up to $10.92 \mathrm{Gbits} / \mathrm{s}$, logic utilization does not exceed $1.25 \%$ and flip-flops utilization is less than $0.45 \%$. Extending to 2-D and 3-D multiscrolls increases the number of distinct output streams from 8 to 64 and 512 respectively because of 3

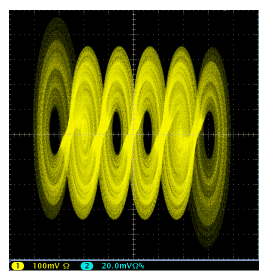

(a)

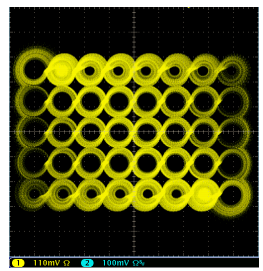

(d)

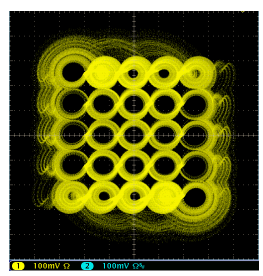

(g)

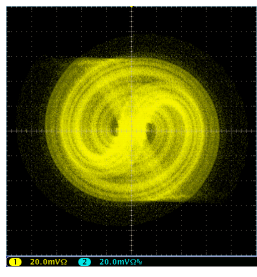

(b)

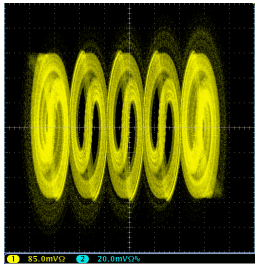

(e)

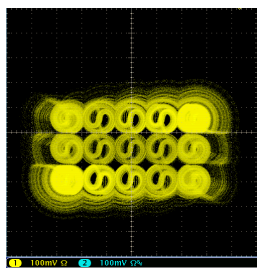

(h)

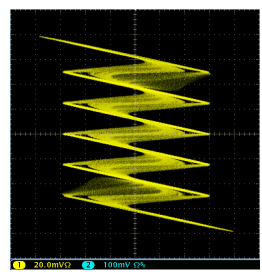

(c)

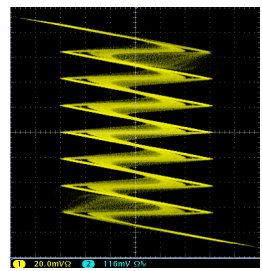

(f)

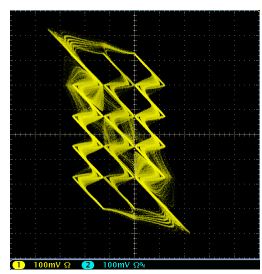

(i)
Fig. 4. Oscilloscope trace of experimentally obtained X-Y, Y-Z and Z-X attractors from the digitally implemented (a)-(c) 1-D chaos with $U_{x}=-1$ [6 scrolls], (d)-(f) 2-D chaos with $\left(U_{x}, U_{y}\right)=(1,-2)[8 \times 5$ scrolls] and (g)-(i) 3-D chaos with $\left(U_{x}, U_{y}, U_{z}\right)=(-2,-2,-4)[5 \times 5 \times 3$ scrolls].
TABLE II

NIST SP. 800-22 TEST RESULTS AND AREA/PERFORMANCE RESULTS ON XILINX VIRTEX 4 FPGA.

\begin{tabular}{|c|c|c|c|c|c|c|}
\hline \multicolumn{7}{|c|}{ NIST SP. $800-22$ Results } \\
\hline & \multicolumn{2}{|c|}{$1-\mathrm{D}$} & \multicolumn{2}{|c|}{$2-\mathrm{D}$} & \multicolumn{2}{|c|}{$3-\mathrm{D}$} \\
\hline & PV & $\mathrm{PP}$ & PV & $\mathrm{PP}$ & PV & $\mathrm{PP}$ \\
\hline Monobits & $\checkmark$ & 0.99 & $\checkmark$ & 1.00 & $\checkmark$ & 0.97 \\
\hline Block Frequency & $\checkmark$ & 1.00 & $\checkmark$ & 1.00 & $\checkmark$ & 1.00 \\
\hline Cumulative Sum & $\checkmark$ & 0.99 & $\checkmark$ & 0.99 & $\checkmark$ & 0.99 \\
\hline Runs & $\checkmark$ & 0.99 & $\checkmark$ & 0.96 & $\checkmark$ & 0.97 \\
\hline Longest Run & $\checkmark$ & 1.00 & $\checkmark$ & 0.99 & $\checkmark$ & 1.00 \\
\hline Rank & $\checkmark$ & 1.00 & $\checkmark$ & 0.99 & $\checkmark$ & 0.99 \\
\hline FFT & $\checkmark$ & 0.99 & $\checkmark$ & 1.00 & $\checkmark$ & 1.00 \\
\hline N. O. Temp. & $\checkmark$ & 0.99 & $\checkmark$ & 0.99 & $\checkmark$ & 0.99 \\
\hline O. Temp. & $\checkmark$ & 0.99 & $\checkmark$ & 0.99 & $\checkmark$ & 1.00 \\
\hline Universal & $\checkmark$ & 0.99 & $\checkmark$ & 0.99 & $\checkmark$ & 0.94 \\
\hline Approx. Entropy & $\checkmark$ & 0.94 & $\checkmark$ & 0.96 & $\checkmark$ & 0.99 \\
\hline Random Excursion & $\checkmark$ & 0.98 & $\checkmark$ & 1.00 & $\checkmark$ & 0.98 \\
\hline Random Excursion V. & $\checkmark$ & 0.99 & $\checkmark$ & 0.99 & $\checkmark$ & 0.98 \\
\hline Serial & $\checkmark$ & 0.99 & $\checkmark$ & 0.99 & $\checkmark$ & 0.99 \\
\hline Linear Complexity & $\checkmark$ & 1.00 & $\checkmark$ & 0.99 & $\checkmark$ & 1.00 \\
\hline \multicolumn{7}{|c|}{ Experimental Results on the XC4VSX35-10FF668 FPGA } \\
\hline RNG Bits & & & & & & \\
\hline Frequency [MHz] & & & & 63 & & .04 \\
\hline Throughput $[\mathrm{Gb} / \mathrm{s}]$ & & & & & & \\
\hline Total LUTs & & & & & & \\
\hline Total Flip-Flops & & & & & & \\
\hline
\end{tabular}

additional control bits in $\mathrm{Y}$ and Z. 2-D and 3-D systems provide additional security due to increased nonlinear complexity (observed by higher MLE) at an area and performance cost. Table III summarizes the statistics of the absolute value of cross-correlation coefficients from the 8,64 and 512 distinct output bitstreams from 1-D, 2-D and 3-D multiscroll chaos and each resulting spreading sequence. On average, truncation of defective bits suppresses cross-correlation by a factor of 10 , with the minimum reported as $2.80 \times 10^{-9}$. 3-D multiscroll chaos can support the most users with 512 modes.

\section{Multiple Access Communications}

Modulation with different control parameters guarantees that each user exploits a different effective nonlinearity while all users have the same circuit. Significantly, this does not rely on initial condition sensitivity or different individual generators [3], [4] while also providing complex 3-D dynamics

TABLE III

CROSS-CORRELATION STATISTICS BETWEEN OUTPUTS FOR DIFFERENT CONTROL PARAMETERS FOR 1-D, 2-D AND 3-D MULTISCROLL CHAOS AND CORRESPONDING SPREADING SEQUENCES.

\begin{tabular}{cc|ccccc}
\hline & & Max & Min & Mean & Median & Std. Dev. \\
\hline 1-D & X & 0.1678 & 0.00175 & 0.0364 & 0.0241 & 0.03806 \\
& Y & 0.0169 & 0.00065 & 0.0066 & 0.0064 & 0.00418 \\
& Z & 0.0178 & 0.00052 & 0.0073 & 0.0062 & 0.00495 \\
& SS & $\mathbf{0 . 0 0 2 9}$ & $\mathbf{7 . 2 2 e - 0 6}$ & $\mathbf{0 . 0 0 1 4}$ & $\mathbf{0 . 0 0 1 6}$ & $\mathbf{0 . 0 0 0 7 4}$ \\
\hline 2-D & X & 0.0521 & $4.37 \mathrm{e}-05$ & 0.0194 & 0.0201 & 0.01077 \\
& Y & 0.0630 & $3.52 \mathrm{e}-05$ & 0.0178 & 0.0153 & 0.01279 \\
& Z & 0.0208 & $1.37 \mathrm{e}-07$ & 0.0047 & 0.0039 & 0.00352 \\
& SS & $\mathbf{0 . 0 0 5 6}$ & $\mathbf{3 . 7 0 e - 0 8}$ & $\mathbf{0 . 0 0 1 3}$ & $\mathbf{0 . 0 0 1 1}$ & $\mathbf{0 . 0 0 0 9 6}$ \\
\hline $3-D$ & X & 0.0495 & $5.94 \mathrm{e}-07$ & 0.0145 & 0.0139 & 0.00864 \\
& Y & 0.0695 & $7.99 \mathrm{e}-08$ & 0.0171 & 0.0152 & 0.01165 \\
& Z & 0.1275 & $4.02 \mathrm{e}-07$ & 0.0251 & 0.0212 & 0.02009 \\
& SS & $\mathbf{0 . 0 0 8 1}$ & $\mathbf{2 . 8 0 e - 0 9}$ & $\mathbf{0 . 0 0 1 3}$ & $\mathbf{0 . 0 0 1 1}$ & $\mathbf{0 . 0 0 0 9 5}$ \\
\hline
\end{tabular}


for higher security compared to 1-D maps. Potentially, the utilization of different initial conditions could also provide an additional layer of security.

\section{A. System Description and Performance Analysis}

The $i$-th user transmits the binary symbol sequence $\left\{d_{l}^{(i)}\right\}$ (values of \pm 1 ) using the chaotic spreading sequence $\left\{x_{k}^{(i)}\right\}$ with a spreading factor of $\beta$ that denotes the number of chaotic samples transmitted for a single symbol (the $l$-th bit duration). Therefore, the transmitted signal from the $i$-th user $\left\{s_{k}^{(i)}\right\}$ and the received signal $r_{k}$ can be written as:

$$
\begin{gathered}
s_{k}^{(i)}=d_{l}^{(i)} x_{k}^{(i)}, \quad k \in(\beta(l-1), \beta l] \\
r_{k}=\sum_{i=1}^{M} s_{k}^{(i)}+n_{k}
\end{gathered}
$$

The received signal consists of the sum of all transmitted signals (assuming they are all synchronized) with the AWGN of zero mean and variance $N_{0} / 2$. Since the chaotic samples $\left\{x_{k}^{(i)}\right\}$ are regenerated exactly at the receiver, synchronization is assumed [5]. A correlation-based receiver [6] is used and the decoded symbol $\widetilde{d}^{(j)}$ for the $j$-th receiver is given by:

$$
w_{l}^{(j)}=\sum_{k=1+\beta(l-1)}^{l \beta} r_{k} x_{k}^{(j)}, \widetilde{d}^{(j)}=\operatorname{sgn}\left(w_{l}^{(j)}\right)
$$

Since $\left\{x_{k}^{(i)}\right\}$ only takes values of \pm 1 , fully passes NIST statistical tests and has low cross-correlation between users:

$$
\begin{gathered}
E\left[x_{k}\right] \approx 0, E\left[x_{k} x_{m}\right] \approx 0, \quad k \neq m \\
\operatorname{var}\left[x_{k}\right]=E\left[x_{k}^{2}\right]-E\left[x_{k}\right]^{2}=1 \\
\operatorname{cov}\left[x_{k}^{2}, x_{m}^{2}\right]=E\left[x_{k}^{2} x_{m}^{2}\right]-E\left[x_{k}^{2}\right] E\left[x_{m}^{2}\right]=0 \forall k, m
\end{gathered}
$$

Using the Gaussian approximation [4], the expression for the bit-error rate (BER) at the $j$-th receiver is:

$$
\begin{gathered}
\operatorname{BER}^{(j)}=\frac{1}{2} \operatorname{erfc}\left(\left[2(M-1) / \beta+E_{b} / N_{0}\right]^{-\frac{1}{2}}\right) \\
\operatorname{erfc}(\phi)=\frac{1}{\sqrt{\pi}} \int_{\phi}^{\infty} \exp \left(-\tau^{2}\right) d \tau
\end{gathered}
$$

where $E_{b}=\beta$ is the energy per bit and is normalized such that all users are transmitting at the same average power. Binary-valued spreading code guarantees that $\operatorname{var}\left[x_{k}\right]=$ $\operatorname{cov}\left[x_{k}^{2}, x_{m}^{2}\right]=0$, ensuring a constant bit energy for each user, and low correlations for different bitstreams even with finite length, thus optimizing BER [4]. Fig. 5(a) shows the BER as a function of $E_{b} / N_{0}$ for a 4-user system with different $\beta$. The solid curve denoting the single-user case is shown for reference. BER performance improves for higher $\beta$ and degrades for higher $M$ as expected. Fig. 5(b) shows BER against number of users at $E_{b} / N_{0}=10 \mathrm{~dB}$ for different $\beta$. Correspondence between the theoretical BER and brute-force simulations indicates that the approximations in (8) are valid.

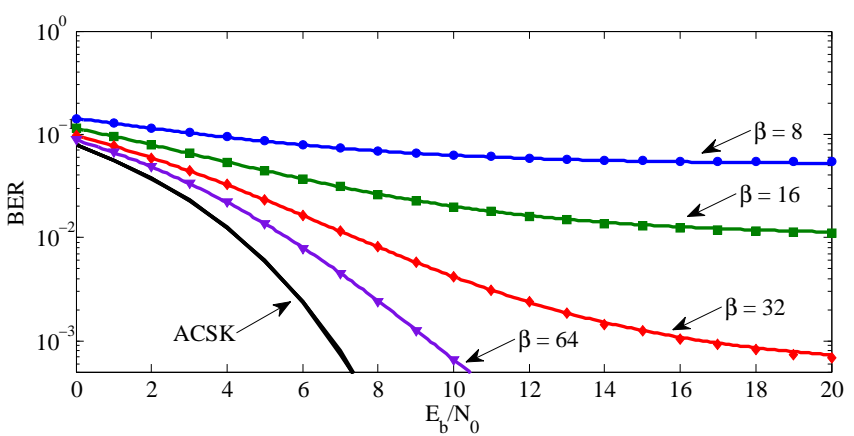

(a)

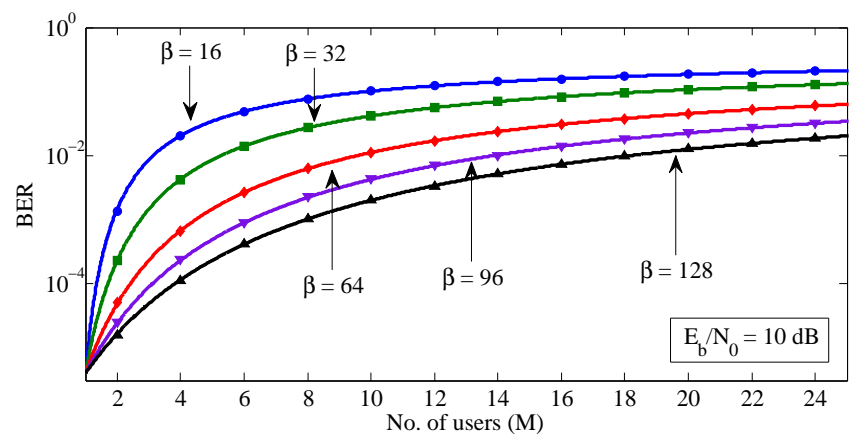

(b)

Fig. 5. (a) BER v/s $E_{b} / N_{0}$ for a 4-user system and single user antipodal CSK (ACSK) under AWGN for different $\beta$ using the 3-D multiscroll pseudonoise and (b) BER v/s number of users for different $\beta$. Markers indicate simulation results while lines indicate theoretical results.

\section{B. Resistance to Multipath Effects}

The proposed spreading codes have favorable delta-like autocorrelation, shown in Fig. 6, indicating that delayed versions of the received signal will appear uncorrelated. Multipath performance analysis is proposed in [8] where the received signal for one bit duration is:

$$
r_{k}=\sum_{i=1}^{M} \sum_{p=0}^{P_{i}} C_{p}^{(i)} s_{k-k_{p}^{(i)}}^{(i)}+n_{k}, \sum_{p=0}^{P_{i}}\left|C_{p}^{(i)}\right|^{2}=1
$$

with $\left\{s_{k}^{(i)}\right\}$ specified by (6a) while $C_{p}^{(i)}$ and $k_{p}^{(i)}$ represent filter coefficients and delays for path $\mathrm{p}$ of the $\mathrm{i}$-th transmitter. All $k_{p}^{(i)}$ are integer multiples of the chaotic sample time and a RAKE receiver with perfect channel estimation is used. 6-path channel for each user is illustrated with each path having the same power (i.e. equal $C_{p}$ 's), giving the impulse response of the channel as:

$$
h_{k}=6^{-1 / 2}\left(\delta_{k}+\delta_{k-1}+\delta_{k-2}+\delta_{k-3}+\delta_{k-4}+\delta_{k-5}\right)
$$

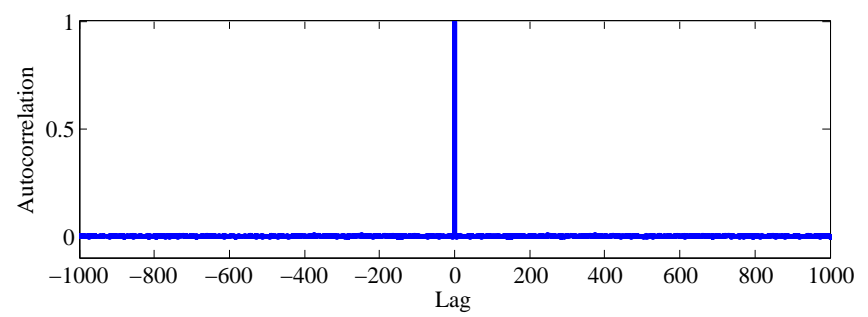

Fig. 6. Autocorrelation of the proposed spreading code from the 3-D multiscroll system. 


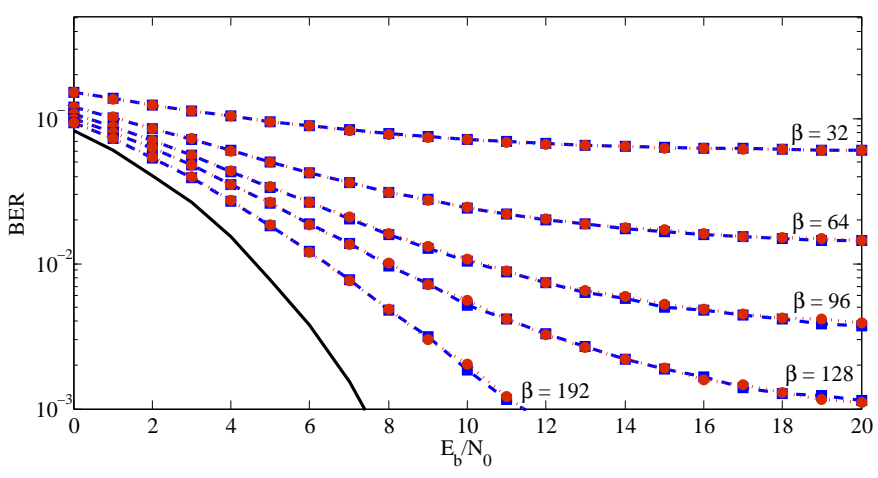

Fig. 7. BER performance of 3-D multiscroll chaotic spreading code and Gold codes in AWGN and multipath environments for different $\beta$. Square markers represent chaotic codes and round markers represent Gold codes.

Fig. 7 shows the BER performance of the proposed spreading code compared to Gold codes for different code lengths and with $M=4$. The strong correspondence indicates that the proposed chaotic codes can provide a security enhancement without any compromise in BER performance.

\section{CONCLUSION}

A generalized hardware implementation of fully digital multidimensional multiscroll chaos through the sawtooth nonlinearity. 1-D, 2-D and 3-D multiscroll chaos is realized using a pipelined architecture and the Euler approximation with chaotic behavior indicated through positive maximum Lyapunov exponent. Furthermore, the number of scrolls in all three dimensions can be manipulated in real-time through a controllable upper bound to give attractors with different effective nonlinearities. Low-significant bits from each dimension are concatenated to yield 512 uncorrelated output streams that pass NIST SP. 800-22 tests. Application as spreading code in a direct sequence CDMA system is emphasized in AWGN and multipath environments, showing performance equivalent to Gold codes. In general, our system offers the same advantages as conventional codes but provides much higher security with easy hardware implementation, as shown by our functional FPGA implementation and with throughput up to 10.92 Gbits/s.

\section{REFERENCES}

[1] A. Abel and W. Schwarz, "Chaos communications-principles, schemes, and system analysis," Proceedings of the IEEE, vol. 90, no. 5, pp. 691710,2002

[2] F. C. M. Lau, M. M. Yip, C. K. Tse, and S. F. Hau, "A multiple-access technique for differential chaos-shift keying," IEEE Transactions on Circuits and Systems I: Fundamental Theory and Applications, vol. 49, no. 1, pp. 96-104, 2002.

[3] M. Long, Y. Chen, and F. Peng, "Simple and accurate analysis of ber performance for dcsk chaotic communication," IEEE Communications Letters, vol. 15, no. 11, pp. 1175-1177, 2011.

[4] W. M. Tam, F. C. M. Lau, C. K. Tse, and A. J. Lawrance, "Exact analytical bit error rates for multiple access chaos-based communication systems," IEEE Transactions on Circuits and Systems II: Express Briefs, vol. 51, no. 9, pp. 473-481, 2004.

[5] G. Mazzini, G. Setti, and R. Rovatti, "Chaotic complex spreading sequences for asynchronous DS-CDMA-part I: system modeling and results," IEEE Transactions on Circuits and Systems I: Fundamental Theory and Applications, vol. 44, no. 10, pp. 937-947, 1997.
[6] M. Sushchik, L. S. Tsimring, and A. R. Volkovskii, "Performance analysis of correlation-based communication schemes utilizing chaos," IEEE Transactions on Circuits and Systems I: Fundamental Theory and Applications, vol. 47, no. 12, pp. 1684-1691, 2000.

[7] G. Mazzini, R. Rovatti, and G. Setti, "Chaos-based asynchronous DSCDMA systems and enhanced rake receivers: Measuring the improvements," IEEE Transactions on Circuits and Systems I: Fundamental Theory and Applications, vol. 48, no. 12, pp. 1445-1453, 2001.

[8] G. Kaddoum, D. Roviras, P. Chargé, and D. Fournier-Prunarety, "Performance of multi-user chaos-based DS-CDMA system over multipath channel," IEEE International Symposium on Circuits and Systems (ISCAS'09), pp. 2637-2640, 2009.

[9] M. E. Yalcin, J. A. K. Suykens, and J. Vandewalle, "Experimental confirmation of 3-and 5-scroll attractors from a generalized Chua's circuit," IEEE Transactions on Circuits and Systems I: Fundamental Theory and Applications, vol. 47, no. 3, pp. 425-429, 2000.

[10] M. E. Yalcin, S. Ozoguz, J. A. K. Suykens, and J. Vandewalle, "n-scroll chaos generators: A simple circuit model," Electronics Letters, vol. 37, no. 3, pp. 147-148, 2001.

[11] J. Lü, F. Han, X. Yu, and G. Chen, "Generating 3-D multi-scroll chaotic attractors: A hysteresis series switching method," Automatica, vol. 40, no. 10 , pp. $1677-1687,2004$

[12] S. Yu, J. Lu, W. K. S. Tang, and G. Chen, "A general multiscroll lorenz system family and its realization via digital signal processors," Chaos, vol. 16, no. 3, p. 033126, 2006.

[13] S. Ozoguz, A. S. Elwakil, and K. N. Salama, "N-scroll chaos generator using nonlinear transconductor," Electronics Letters, vol. 38, no. 14, pp. 685-686, 2002.

[14] M. A. Zidan, A. G. Radwan, and K. N. Salama, "Controllable vshape multiscroll butterfly attractor: System and circuit implementation," International Journal of Bifurcation and Chaos, vol. 22, no. 6, p. 1250143, 2012

[15] K. N. Salama, S. Ozoguz, and A. S. Elwakil, "Generation of n-scroll chaos using nonlinear transconductors," IEEE International Symposium on Circuits and Systems (ISCAS'03), vol. 3, pp. 176-179, 2003.

[16] J. Lü and G. Chen, "Generating multiscroll chaotic attractors: theories, methods and applications," International Journal of Bifurcation and Chaos, vol. 16, no. 4, pp. 775-858, 2006.

[17] A. G. Radwan, A. M. Soliman, and A. S. Elwakil, "1-D digitallycontrolled multiscroll chaos generator," International Journal of Bifurcation and Chaos, vol. 17, no. 1, pp. 227-242, 2007.

[18] A. S. Mansingka, A. G. Radwan, M. A. Zidan, and K. N. Salama, "Analysis of bus width and delay on a fully digital signum nonlinearity chaotic oscillator," IEEE International Midwest Symposium on Circuits and Systems (MWSCAS'11), pp. 1-4, 2011.

[19] J. C. Sprott, "Simple chaotic systems and circuits," American Journal of Physics, vol. 68, no. 8, pp. 758-763, 2000.

[20] M. A. Zidan, A. G. Radwan, and K. N. Salama, "Random number generation based on digital differential chaos," IEEE International Midwest Symposium on Circuits and Systems (MWSCAS'11), pp. 1-4, 2011.

[21] A. S. Mansingka, A. G. Radwan, and K. N. Salama, "Fully digital 1D, 2-D and 3-D multiscroll chaos as hardware pseudo random number generators," IEEE International Midwest Symposium on Circuits and Systems (MWSCAS'12), pp. 1180-1183, 2012.

[22] M. A. Zidan, A. G. Radwan, and K. N. Salama, "The effect of numerical techniques on differential equation based chaotic generators," IEEE International Conference on Microelectronics (ICM'11), pp. 1-4, 2011.

[23] F. Agnelli, G. Mazzini, R. Rovatti, and G. Setti, "A first experimental verification of optimal MAI reduction in chaos-based DS-CDMA systems," IEEE International Symposium on Circuits and Systems (ISCAS'01), vol. 3, pp. 137-140, 2001.

[24] I. Martoyo, A. Susanto, E. Wijanto, H. Kanalebe, K. Gandi et al., "Chaos codes vs. orthogonal codes for CDMA," IEEE International Symposium on Spread Spectrum Techniques and Applications (ISITA'10), pp. 189193, 2010.

[25] A. S. Mansingka, A. G. Radwan, and K. N. Salama, "Design, implementation and analysis of fully digital 1-d controllable multiscroll chaos," IEEE International Conference on Microelectronics (ICM'11), pp. 1-5, 2011.

[26] S. Kodba, M. Perc, and M. Marhl, "Detecting chaos from a time series," European journal of physics, vol. 26, no. 1, pp. 205-215, 2005.

[27] A. Rukhin et. al., "A statistical test suite for random and pseudorandom number generators for cryptographic applications," NIST Special Publication 800-22, 2001. 